\title{
Quality of probiotic products for preterm infants: Contamination and missing strains
}

\author{
Marijn J. Vermeulen ${ }^{1}$ (D) | Ad Luijendijk ${ }^{2}$ | Letty van Toledo ${ }^{3}$ | Anton H. van Kaam ${ }^{3}$ | \\ Irwin K. M. Reiss ${ }^{1}$
}

${ }^{1}$ Division of Neonatology, Department of Paediatrics, Erasmus MC Sophia Children's Hospital, Rotterdam, The Netherlands

${ }^{2}$ Department of Medical Microbiology and Infectious Diseases, Erasmus MC, Rotterdam, The Netherlands

${ }^{3}$ Department of Neonatology, Emma Children's Hospital Amsterdam

UMC, University of Amsterdam, Amsterdam, The Netherlands

Correspondence

Marijn J. Vermeulen, paediatricianneonatologist, Erasmus MC - Sophia Children's Hospital, Office Sp-4462, PO Box 2060, 3000 CB Rotterdam, The Netherlands.

Email: m.j.vermeulen@erasmusmc.nl

\begin{abstract}
Probiotics are effective in reducing necrotising enterocolitis in preterm infants, but routine use is not generally adopted. We describe a safety issue concerning contamination by pathogenic bacteria and missing of labelled strains in a probiotic product widely used in neonatal care. We recommend all centres using probiotics in the care of vulnerable patients to consider product safety checks in addition to the quality reports of manufacturers. Meanwhile, clinicians and manufacturers should collaborate to define quality standards for probiotic products in clinical settings as long as specific international regulations are lacking.
\end{abstract}

\section{KEYWORDS}

contamination, Infloran, necrotising enterocolitis, probiotics, safety
Probiotics can prevent necrotizing enterocolitis (NEC) in preterm infants, reducing the incidence by nearly half in meta-analysis of trials including over 10000 infants. ${ }^{1}$ Still, probiotic use is not generally adopted in all neonatal intensive care units (NICUs), varying from $14 \%$ in the United States to $58 \%$ in Germany. ${ }^{2,3}$ Why is a cost-effective therapy not generally adopted by all neonatal centres worldwide? First, evidence is still lacking in the most optimal treatment strategy regarding the strains and dose. ${ }^{4}$ Second, availability of products actually tested for efficacy in clinical trials is limited. Third, reporting of safety data is often lacking or inadequate. ${ }^{5}$ One concern is the risk of probiotic sepsis, which has been reported in several preterm infant cases. ${ }^{6}$ However, this complication seems to be very rare, not always corresponds to the probiotics in the product, and occurs mainly in infants with additional risk factors compromising gut integrity or in association with congenital anomalies. ${ }^{3,7-9}$ On the other hand, probiotic bacteriaemia may have been overlooked in other cases, because anaerobic strains can be difficult to culture from standard single paediatric culture bottles. Fourth, there may be local regulatory issues on a complex product being marketed as either a drug or a food additive with or without medical purpose. ${ }^{10}$ For example, after 2009 , we faced stringent additional conditions of the Health Inspectorate, after a Dutch probiotic trial in adults with acute pancreatitis showed increased mortality in the intervention group. ${ }^{11}$ Here, we report our experience with another safety issue which has led to ceasing of probiotic administration in our level III NICU: product contamination and missing of labelled strains.

Since 2012, approximately 900 preterm infants were treated with routine probiotics for the prevention of NEC in our level III $\mathrm{NICU}$ of the Erasmus MC in Rotterdam. According to local protocol, we treated all infants with a gestational age below 32 weeks or birthweight below 1500 grams starting from day one. ${ }^{12}$ We used Infloran ${ }^{\circledR} 250 \mathrm{mg}$ capsules, containing a combination of $10^{9} \mathrm{CFU}$ Lactobacillus acidophilus and $10^{9} \mathrm{CFU}$ Bifidobacterium bifidum (SIT Laboratorio Farmaceutico, Mede, Italy), which had shown NEC reduction in a double-blind trial. ${ }^{13}$ We strictly followed all storage and handling procedures. During the treatment period, we observed a significant decline in the incidence of NEC from $7.8 \%$ to $5.1 \%$ (OR 0.63 (95\% Cl 0.42-0.93), $P=.02$ ). The NEC reduction was not significantly associated with the introduction of probiotics after adjustment for potential confounders including the interaction with type of feeding (only breast milk, mixed or only formula) and the underlying downtrend in NEC incidence. ${ }^{12}$ Because of increasing concerns on the quality and safety of probiotics products used in clinical practice, we did routine quality checks on the product content. 
We tested 15 capsules from five different strips, originating from three different lots. The expiry dates ranged from 3-13 months after testing. To detect both aerobic and anaerobic bacteria, fungi and yeasts, we cultured the content on 4 different agar plates for 5 days, according to the following procedures. The full content of probiotic powder of each tested capsule was dissolved in $1 \mathrm{~mL}$ $\mathrm{BHI}$ broth with Vitamin B6. Then, $10 \mu \mathrm{L}$ was plated on TSA blood agar (BA), Chocolate agar ( $\mathrm{CHOC}$ ), Mac Conkey agar (McC) and Hemin and Vitamin K1 enriched Brucella Blood Agar plate (BBA). ${ }^{14}$ Simultaneously, sterility control of the BHI-VitB6 was set. The BA, $\mathrm{CHOC}$ and $\mathrm{McC}$ were incubated for 5 days at $35^{\circ} \mathrm{C}$ and $5 \% \mathrm{CO} 2$, and the BBA was incubated 5 days anaerobically at $35^{\circ} \mathrm{C}$. All grown bacteria were identified by MALDI-TOF (Bruker). In only 1 lot purchased, both labelled probiotic microorganisms were detectable in all capsules. In the other two lots, one or both strains were missing and varying contamination was found, with Streptococcus oralis, Lactococcus garvieae, Enterococcus feacalis, Enterococcus faecium and Lactococcus lactis (Table 1).

The level III NICU of the Emma Children's Hospital in Amsterdam was about to start with probiotics in preterm infants and independently purchased the same product. As they also found contamination with $S$ oralis in lot 4583A, we consider contamination after purchase highly unlikely.

Unfortunately, we were not able to perform additional validation or quantitative PCR, because the disapproved lots were directly returned to the manufacturer according to hospital regulations. By plating 1/100 of dissolved full capsules (that are supposed to contain $10^{9}$ CFU of each labelled probiotic microorganisms), one would ideally expect to culture $10^{7} \mathrm{CFUs}$ of each probiotic strain. Based on this estimated detection level, we can assume that the capsules without detectable probiotics contained $<100$ CFU per capsule.

Even without knowing the exact quantity of contaminants, we question the safety of the contaminated products. The possible clinical consequences of a contaminated probiotic product have dramatically been shown in 2014, by a fatal gastrointestinal mucormycosis

TABLE 1 Microorganisms identified in capsules with label claiming Lactobacillus acidophilus and Bifidobacterium bifidum, by MALDI-TOF. Shown are percentages of capsules of the specified lot tested positive for mentioned microorganism

\begin{tabular}{|c|c|c|c|}
\hline Lot & 4307B & $4583 A$ & $5145 \mathrm{~A}$ \\
\hline Capsules tested & $N=4$ & $N=4$ & $N=7$ \\
\hline \multicolumn{4}{|l|}{ Label claimed } \\
\hline Lactobacillus acidophilus & $100 \%$ & $100 \%$ & $0 \%$ \\
\hline Bifidobacterium bifidum & $100 \%$ & $75 \%$ & $0 \%$ \\
\hline \multicolumn{4}{|l|}{ Contaminants } \\
\hline Streptococcus oralis & - & $100 \%$ & - \\
\hline Lactococcus garvieae & - & - & $100 \%$ \\
\hline Enterococcus feacalis & - & - & $57 \%$ \\
\hline Enterococcus faecium & - & - & $43 \%$ \\
\hline Lactococcus lactis & - & - & $29 \%$ \\
\hline
\end{tabular}

in a preterm infant. ${ }^{15}$ We could only find two other reports on contaminated products as a cause of infant sepsis, of whom both have recovered well. ${ }^{16,17}$ Pathogenicity of the contaminating microorganisms is difficult to rule out in preterm infants whose gastrointestinal barrier function and immune system are still compromised. Even apparently commensal skin and gastrointestinal bacteria frequently cause sepsis in very preterm infants. ${ }^{18,19}$ We detected $L$ lactis, which may be harmless as natural exposure is likely common for its widespread use in cheese industry without reports on pathogenicity. ${ }^{20}$ L garviae is associated with outbreaks of infections in fish. It is a rare cause of infective endocarditis in adults with valvular disease after ingestion of raw seafood, with no reports on infection in children. ${ }^{21}$ The human commensals Streptococcus oralis (including Streptococcus pneumoniae) and Enterococcus feacalis and faecium are likely of most concern, as these can cause neonatal sepsis in preterm infants. ${ }^{18}$

The tested probiotic product is produced according to general European drug regulations that lack special regulations for probiotic products. ${ }^{22}$ In accordance to this and other international Pharmacopeas, microbiological quality of 'non-sterile drugs' is deemed acceptable if Escherichia coli, Staphylococcus aureus and Salmonella species are absent, and if total counts of bile-tolerant Gram-negative bacteria, aerobic microbes and yeast or moulds are below predefined levels of colony forming units (CFU) per gram or $\mathrm{mL}^{23,24}$ We suggest that these regulations may not be strict enough when it comes to safety of probiotic use in preterm infants and that redefinition of so-called 'objectionable organisms' for this vulnerable population is needed. From a clinical point of view, an ideal product would contain only the labelled probiotic strains, in an acceptable quantity during shelf life with no contamination of any other microorganism at all.

In response to our report, the manufacturer retested the products and confirmed contamination of lot $4583 \mathrm{~A}$ and 5145A (personal communication). They did not report on the presence or absence of the labelled probiotic strains in the product. As the product was still considered safe according to the regulations, no recall action was taken by the manufacturer. Of the three lots tested, we only used the uncontaminated lot (4307B) in patient care. When products with missing strains have been administered earlier, this may have been for no benefit and may explain the lack of effect on NEC in our timeseries analyses. ${ }^{12}$ We have stopped administration of probiotics in our unit until we receive products that pass our own safety and viability checks.

This report has several limitations, as it is just a description of our local clinical findings on a single product, and not part of a prospective quality control project. In retrospect, we should have quantified the contaminants by plate count or qPCR. Considering the sensitive methods used, bacterial enumeration of the contaminants may have been low, which can limit the clinical relevance. Next, additional testing for non-viable probiotic bacteria could have helped to differentiate between issues in the production process versus limited viability during shelf life. However, the product label claims live probiotics, by declaring the quantitative amount as CFU per capsule. Viability and purity testing would ideally be done along the chain, from production, storage and shipping to the hospital, pharmacy and patient ward, to 
reveal critical steps in the process. However, we consider quality control the responsibility of the manufacturer and regulatory agencies and we lack funding to study this in more detail. How our findings relate to other products and other settings are unclear. Of note, we have no data on the product widely used in Germany, with the same brand name but different content (Bifidobacterium infantis instead of Bifidobacterium bifidum) or on any other products. However, our findings are not unique. Earlier reports have shown that mismatches between the content and the label claims are common in commercial probiotic products. Reported composition mismatches included incorrect taxonomy, presence of un-labelled species or missing of the labelled (sub) species. Similar to our findings, these content biases were found with pill-to-pill (within a lot) and lot-to-lot variation. ${ }^{25,26}$ Another possible shortcoming is that we have no access to detailed quality control findings of the manufacturer. The main strengths of this report include our methods to detect a wide range of potential contaminants. Methods were based on international standards with additional state-of-the-art culture and detection procedures, run in a ISO 15189 certified laboratory. The contamination by S oralis was confirmed by an independent laboratory, and the positive findings in the first lot indicate sufficient sensitivity to detect the probiotics.

We aim to call attention to probiotic product quality, as these can threat patient safety in at-risk populations, but may also lead to bias and misinterpretation of clinical trial results. This underlines the need for independent quality control, and for rapid and accurate molecular techniques for identification of (sub) species, such as quantitative real-time PCR, flow cytometry and MALDI-TOF Mass Spectrometry. ${ }^{24,27}$ However, also by simple culture techniques, contamination of products may be found.

In conclusion, despite the mass of current literature showing that probiotics can prevent NEC and save many lives, general adoption of probiotic use is hampered by different issues. This report illustrates that quality issues of the products currently available are one of them. We are reluctant to withhold patients a highly effective treatment, but we are also worried about the potential risks of impure products. We understand that quality control of unsterile products is complex and that $100 \%$ pure products are difficult to guarantee. As long as regulatory requirements may not be strict enough, we recommend all centres using probiotics in the care of vulnerable patients to consider routine bacterial checks in addition to quality checks of manufacturers to guarantee product safety. The same warning holds true for ongoing and future clinical trials. Meanwhile, clinicians, microbiologists and manufacturers should collaborate to define population-specific quality standards for probiotic products in clinical settings as long as specific international regulations are lacking for at-risk populations.

\section{CONFLICT OF INTEREST}

None of the authors have anything to disclose regarding potential/perceived conflicts of interest or financial ties to products in the study. MJV is unpaid Scientific Advisory Board member of Neobiomics (www.neobiomics.eu).
ORCID

Marijn J. Vermeulen (iD https://orcid.org/0000-0001-9686-8186

\section{REFERENCES}

1. Patel RM, Underwood MA. Probiotics and necrotizing enterocolitis. Semin Pediatr Surg. 2018;27:39-46.

2. Viswanathan S, Lau C, Akbari H, Hoyen C, Walsh MC. Survey and evidence based review of probiotics used in very low birth weight preterm infants within the United States. J Perinatol. 2016;36:1106-1111.

3. Denkel LA, Schwab F, Garten L, Geffers C, Gastmeier P, Piening B. Protective effect of dual-strain probiotics in preterm infants: a multicenter time series analysis. PLoS ONE. 2016;11:e0158136.

4. van den Akker CHP, van Goudoever JB, Szajewska H, et al. Probiotics for preterm infants: a strain specific systematic review and network meta-analysis. J Pediatr Gastroenterol Nutr. 2018;67(1):103-122.

5. Bafeta A, Koh M, Riveros C, Ravaud P. Harms reporting in randomized controlled trials of interventions aimed at modifying microbiota: a systematic review. Ann Intern Med. 2018;169:240-247.

6. Costa RL, Moreira J, Lorenzo A, Lamas CC. Infectious complications following probiotic ingestion: a potentially underestimated problem? A systematic review of reports and case series. BMC Complement Altern Med. 2018;18:329.

7. Kunz AN, Noel JM, Fairchok MP. Two cases of Lactobacillus bacteremia during probiotic treatment of short gut syndrome. J Pediatr Gastroenterol Nutr. 2004;38:457-458.

8. Ohishi A, Takahashi S, Ito Y, et al. Bifidobacterium septicemia associated with postoperative probiotic therapy in a neonate with omphalocele. J Pediatr. 2010;156:679-681.

9. Esaiassen E, Cavanagh P, Hjerde E, Simonsen GS, Stoen R, Klingenberg C. Bifidobacterium longum Subspecies infantis Bacteremia in 3 extremely preterm infants receiving probiotics. Emerg Infect Dis. 2016;22:1664-1666.

10. Sanders ME, Merenstein DJ, Ouwehand AC, et al. Probiotic use in at-risk populations. J Am Pharm Assoc. 2003;2016(56):680-686.

11. Besselink MGH, van Santvoort HC, Buskens E, et al. Probiotic prophylaxis in predicted severe acute pancreatitis: a randomised, double-blind, placebo-controlled trial. Lancet. 2008;371:651-659.

12. Samuels N, van de Graaf R, Been JV, et al. Necrotising enterocolitis and mortality in preterm infants after introduction of probiotics: a quasi-experimental study. Sci Rep. 2016;6:31643.

13. Lin $\mathrm{HC}$, Hsu CH, Chen $\mathrm{HL}$, et al. Oral probiotics prevent necrotizing enterocolitis in very low birth weight preterm infants: a multicenter, randomized, controlled trial. Pediatrics. 2008;122:693-700.

14. Versalovic J, Carroll KC, Funke G, Jorgensen JH, Landry ML, Warnock DW. Manual of Clinical Microbiology, 10th edn. Washington, DC: American Society for Microbiology; 2011:1. ISBN 978-1-55581-463-2.

15. Vallabhaneni S, Walker TA, Lockhart SR, et al. Notes from the field: fatal gastrointestinal mucormycosis in a premature infant associated with a contaminated dietary supplement-Connecticut, 2014. MMWR Morb Mortal Wkly Rep. 2015;64:155-156.

16. Barton LL, Rider ED, Coen RW. Bacteremic infection with Pediococcus: vancomycin-resistant opportunist. Pediatrics. 2001:107:775-776.

17. Brecht M, Garg A, Longstaff K, Cooper C, Andersen C. Lactobacillus sepsis following a laparotomy in a preterm infant: a note of caution. Neonatology. 2016;109:186-189.

18. Stoll BJ, Hansen N, Fanaroff AA, et al. Late-onset sepsis in very low birth weight neonates: the experience of the NICHD Neonatal Research Network. Pediatrics. 2002;110:285-291. 
19. Downey LC, Smith PB, Benjamin DK Jr. Risk factors and prevention of late-onset sepsis in premature infants. Early Hum Dev. 2010;86(Suppl 1):7-12.

20. Song AA, In L, Lim S, Rahim RA. A review on Lactococcus lactis: from food to factory. Microb Cell Fact. 2017;16:55.

21. Malek A, De la Hoz A, Gomez-Villegas SI, Nowbakht C, Arias CA. Lactococcus garvieae, an unusual pathogen in infective endocarditis: case report and review of the literature. BMC Infect Dis. 2019;19:301.

22. European Directorate for the Quality of Medicines. The European Pharmacopoeia: Microbiological quality of non-sterile pharmaceutical preparations and substances for pharmaceutical use, 10th edn. Strasbourg, France: The European Pharmacopoeia; 2019:627-628.

23. World Health Organization. The International Pharmacopeia, 4th edn. S.3.7 ed2012.

24. Jackson SA, Schoeni JL, Vegge C, et al. Improving end-user trust in the quality of commercial probiotic products. Front Microbiol. 2019;10:739.
25. Lewis ZT, Shani G, Masarweh CF, et al. Validating bifidobacterial species and subspecies identity in commercial probiotic products. Pediatr Res. 2016;79:445-452.

26. Morovic W, Hibberd AA, Zabel B, Barrangou R, Stahl B. Genotyping by PCR and high-throughput sequencing of commercial probiotic products reveals composition biases. Front Microbiol. 2016;7:1747.

27. Angelakis E, Million M, Henry M, Raoult D. Rapid and accurate bacterial identification in probiotics and yoghurts by MALDI-TOF mass spectrometry. J Food Sci. 2011;76:M568-M572.

How to cite this article: Vermeulen MJ, Luijendijk A, van Toledo L, van Kaam AH, Reiss IKM. Quality of probiotic products for preterm infants: Contamination and missing strains. Acta Paediatr. 2019;00:1-4. https://doi.org/10.1111/ apa.14976 